\title{
Simulated Experiential Learning Activity to Empower Paid and Unpaid Caregivers in Dementia Care
}

\author{
Brenda J Gamble* \\ Faculty of Health Sciences, Ontario Tech University, Ontario, Canada
}

*Corresponding author: Brenda J Gamble, Faculty of Health Sciences, Ontario

Tech University, Ontario, Canada.

Received Date: January 19, 2021

Published Date: February 01, 2021

\begin{abstract}
The number of people with dementia is rising worldwide. People with dementia are challenged by the symptoms of their illness, as well by discriminatory attitudes and actions. However, this stigmatization is not only experienced by people with dementia; it is also experienced by the paid and unpaid caregivers who provide care for people with dementia. Education is a key strategy to reduce stigma and improve the quality of life of individuals with dementia. It also has the potential to provide caregivers with meaningful forms of support. A systematic review of the literature demonstrated that the most effective educational intervention to change attitudes and reduce stigma are resources that incorporate an in person contact approach. Dementia Live ${ }^{T M}$ is a simulation tool that places learners "in the shoes" of people with dementia and is used to raise awareness of what it might be like to live with dementia. The targeted population includes students, health care and social care workers, staff from hospitals, longterm care residences, retirement homes, and home care services, as well as friends, volunteers and family caregivers. This educational intervention can serve as a model to develop additional simulation tools to reduce all types of stigma to support a safe learning and work environment.
\end{abstract}

Keywords: Dementia; Stigma; Experiential learn; simulation
Abbreviations:
ELA: Experiential Learning Activity
PWD: People with Dementia
Paid caregivers: Health and Social Care Workers
Unpaid caregivers: Family, Friends and Volunteers

\section{Introduction}

A significant demographic shift is occurring world-wide, resulting in the proportion of people over 60 years of age nearly doubling by 2050 from 12 to $22 \%$ [1]. The aging population is not homogenous. Many older adults will enjoy a full and active life in old age, while others will experience declines in physical and mental health. Dementia is one of the major causes of disability and dependency among older adults [2]. Worldwide it is estimated that the number of people with dementia (PWD) will be 82 million in 2030, and 152 million in 2050 [2]. It is also estimated that double the number of people (i.e. 164 and 304 million) will be indirectly

affected by dementia [2]. This number includes both health/ social care workers (i.e. paid caregivers) and family, friends and volunteers (i.e., unpaid caregivers).

PWD are challenged by both the symptoms of their illness and discriminatory attitudes and actions of family members, friends, caregivers, and society [3]. Stigmatization associated with dementia is also experienced by paid and unpaid caregivers supporting PWD [4]. The stigma associated with dementia prevents people from seeking help [5]. This is true for both PWD and their paid and unpaid caregivers. Reducing the discriminatory attitudes towards 
PWD can potentially contribute to establishing an environment that inspires healthy aging and supports an optimal quality of life for those living with dementia. Additionally, addressing discrimination about dementia will enable paid and unpaid caregivers to provide meaningful support to those in their care.

\section{Discussion}

Education is one of the key strategies to reduce stigma [6]. Livingston, Milne, Fang, and Amari [7] conducted a systematic review of the literature that revealed the most effective approach to reducing stigma are educational strategies that incorporate in-person contact and experience. Ontario Tech University, a certified age friendly university, is committed to teaching, learning and leading-edge research that will help find new and innovative approaches to dementia care.

\section{Experiential learning activity}

A simulated experiential learning activity (ELA) [8] was developed and implemented to address the stigma associated with dementia. The targeted population includes students, health care and social care workers, staff from hospitals, long-term care residences, retirement homes, and home care services, as well as friends, volunteers and family caregivers. The experiential learning approach adopted, incorporates classroom-based simulation to simulate in person contact and experience.

The tool, Dementia Live TM [9], provides individuals with the opportunity to engage in a simulation that exposes learners to the impact of dementia symptoms (e.g., cognitive impairment and sensory changes) on activities of daily living. During this simulation, participants gain a first-hand understanding of the anxiety, isolation, frustration, and confusion that people with dementia live with every day. While the simulation is part of the learning activity, additional elements are needed to enhance learning and encourage reflection on the experience.

The Charles, Bainbridge and Gilbert [10] educational model of exposure, immersion and mastery was adapted to inform the parameters of the simulated ELA. During the exposure phase, learners are provided with information and the facts related to dementia (e.g., definition, numbers of PWD, symptoms, impact of dementia on individuals, health and social care systems and society, etc.). The simulation tool [9] is used in the immersion phase. The overall goal of the simulation is for people to experience what it is like to complete an easy everyday task with altered vision, hearing, and tactile ability, all characteristics of people living with dementia. Debriefing is used in the mastery phase to promote learner reflection and feedback to enhance the learning experience [11].

The simulated ELA has been adapted for continuing education, graduate and undergraduate training using a variety of delivery modes including in person, hybrid and online teaching. As well, partnerships have been established with a variety of communitybased organizations to make the simulated ELA accessible to informal caregivers.

\section{Conclusion}

Dementia Live TM places learners "in the shoes" of PWD to raise awareness of what it might be like to live with dementia. Reducing the discriminatory attitudes towards PWD can contribute to establishing an environment that inspires healthy aging and supports an optimal quality of life for those living with dementia. Activities are currently underway to implement both formative and summative evaluations of the simulated ELA for learners. As well, an evaluation of the experiential learning activity, including the simulation, is being conducted. Why? Stigmatizing attitudes can vary ethically and culturally. For example, gender is an important consideration when examining the impact of stigmatization associated with dementia. It is primarily women who care for and support PWD—be it through paid work [12] or unpaid work [13].

Subsequently, the next steps will be directed at incorporating elements into the design of the experiential learning activity that addresses diversity and equity. Additional work will be undertaken to further evaluate the learning experience in different settings and populations. This will enable us to modify and adapt the simulated ELA as we move forward with the implementation of the experiential learning activity within different contexts and groups. This experiential learning activity has the potential to serve as a model to support a safe learning and work environment and thus, it is an important educational tool.

\section{Acknowledgement}

Dementia Live TM: https://ageucate.com/index.php?main_ page $=$ dementia_live

Funding was provided by: Ontario Tech University, Ontario Canada

Michelle Aaron: Continuing Education Ontario Tech University

Dr. Cathereine Drea, Director Learning Innovations, Ontario Tech University, Ontario Canada

Dr. Wendy Stanyon, Faculty of Health Sciences, Ontario Tech University

\section{Conflict of Interest}

None to declare.

\section{References}

1. WHO: World Health Organization (2018) Ageing and World Health.

2. WHO: World Health Organization (2020) Dementia.

3. Ahmedani BK (2011) Mental Health Stigma: Society, Individuals, and the Profession. J Soc Work Values Ethics 8(2): 41-416.

4. Liu Megan Fong (2011) Perceived stigma in caregivers of persons with dementia and its impact on depressive symptoms. Thesis, University of Iowa, USA.

5. Benbow, Susan Mary, David Jolley (2012) Dementia: Stigma and Its Effects. Neurodegenerative Disease Management 2(2): 165-172.

6. Herrmann Lynn K., Elisabeth Welter, James Leverenz, Alan J Lerner, et al. (2018) A Systematic Review of Dementia-Related Stigma Research: Can We Move the Stigma Dial? Am J Geriatr Psychiatry 26(3): 316-331. 
7. Livingston James D, Teresa Milne, Mei Lan Fang, Erica Amari (2011) The Effectiveness of Interventions for Reducing Stigma Related to Substance Use Disorders: a Systematic Review. Addiction 107(1): 39-50.

8. (2014) Experiential Learning: a Handbook for Education, Training and Coaching. Human Resource Management International Digest 22(2).

9. AGE-u-cate (2021) Experience Dementia Live.

10. Charles Grant, Lesley Bainbridge, John Gilbert (2009) The University of British Columbia Model of Interprofessional Education. J Interprof Care 24(1): 9-18.
11. Palaganas, Janice C, Mary Fey, Robert Simon (2016) Structured Debriefing in Simulation-Based Education. AACN Adv Crit Care 27(1): 78-85.

12. (2019) Gender Equality. Organisation for Economic Co-operation and Development.

13. Sharma Nidhi, Subho Chakrabarti, Sandeep Grover (2016) Gender Differences in Caregiving Among Family-Caregivers of People with Mental Illnesses. World J Psychiatry 6(1): 7-17. 